\title{
UV light generation in optical fibres
}

\author{
Yun Wang ${ }^{1}$, Jing He ${ }^{1}$, M.I.M. Abdul Khudus ${ }^{1}$, Rand Ismaeel ${ }^{1}$, Francesco De Lucia ${ }^{1}$, Pier J. A. Sazio ${ }^{1}$, Peter \\ Horak $^{1}$, Norberto Chiodini ${ }^{2}$, Martynas Beresna ${ }^{1}$, Gilberto Brambilla $^{1}$ \\ ${ }^{1}$ Optoelectronics Research Centre, University of Southampton, Southampton, SO17 1BJ, United Kingdom \\ ${ }^{2}$ Dipartimento di scienza dei materiali, Università di Milano Bicocca, Piazza dell'Ateneo Nuovo, 1 - 20126, Milano, Italy \\ Author e-mail address: G.Brambilla@soton.ac.uk
}

\begin{abstract}
UV light has been generated in optical fibers using nonlinear optics (harmonic generation) and rare earth doping with $\mathrm{Gd}^{3+}$.

OCIS codes: (140.3510) Lasers, fibers, (140.0140) Lasers and laser optics (190.4370) Nonlinear optics, fibers,.
\end{abstract}

\section{Introduction}

Coherent UV light generation has numerous potential applications including lithography, biomedical and explosives detection [1-3]. To date, two main approaches were adopted: 1) the use of nonlinear crystals for harmonic generation of a near IR laser, or 2) excimer gas lasers. These methods use toxic gases, need relatively complicated free space optics, and can have poor beam shape and/or low power. Optical fiber lasers provide a solid state solution with singlemode output beams $\left(\mathrm{M}^{2} \sim 1\right)$. This paper reports two approached pursued for the generation of UV light: the use of rare earth doping (in particular $\mathrm{Gd}^{3+}$ ) in silica optical fibers and fourth and fifth harmonic generation in optical fiber tapers.

\section{Gd-doped optical fibers}

Rare earths have been widely used to achieve near-IR emission in silica fibers, typically with $\mathrm{Yb}$, Er and Tm for emission in the $1-2 \mu \mathrm{m}$ wavelength range. Visible lasers have been achieved using upconversion in Ce or Er doped silica optical fibers, and often required multiple wavelength pumping. Although emission in the UV has been observed for a variety of rare earths, most of the rare earth dopes silica samples decay nonradiatively or through multiphoton emission at longer wavelengths. Bulk silica samples doped with $\mathrm{Gd}^{3+}$ exhibited emission at $\lambda \sim 0.31 \mu \mathrm{m}$ and $\lambda \sim 0.28 \mu \mathrm{m}[4]$ and no multiphoton decay at longer wavelengths [4].

The $\mathrm{Gd}^{3+}$ doped silica optical fibers used in these experiments were fabricated by 1) the modified chemical vapor deposition (MCVD) and solution doping resulting in a pure silica cladding and a phosphosilicate core; and 2) the rod-in-tube technique, resulting in a pure silica core and a fluorosilicare cladding. In both optical fibers pumping in the UV resulted in a strong emission at $\lambda \sim 0.31 \mu \mathrm{m}$.

\section{Harmonic generation}

Harmonic generation has been previously used in tapered optical fibers as a means to convert light from $\lambda=1.5 \mu \mathrm{m}$ to $\lambda=0.5 \mu \mathrm{m}$ [5], but with an efficiency limited by the presence of intrinsic surface waves [6], associated to frozen thermal fluctuations. By combining, second harmonic generation in poled optical fibers with parametric amplification and four wave mixing, generation of light at $\lambda \sim 0.38 \mu \mathrm{m}$ and $\lambda \sim 0.31 \mu \mathrm{m}$ was observed. Importantly, the dependence of the efficiency on the taper diameter was shown to be relatively loose, meaning that the dephasing limitation associated to surface waves could potentially be overcome.

\section{References}

[1] D.S. Moore. Rev. Sci. Instr., 75(8):2499\{2512, 2004.

[2] J. Marshall, et al. Ophthalmology, 92(6):749, 1985.

[3] F. Auzel. Chem. Rev. 104(1):139\{174, 2004.

[4] J. He, Y. Wang, et al., proc. CLEO, JTh2A.86 (2016)

[5] T. Lee, et al., Opt. Expr.20(8):8503, 2012..

[6] M.I.M. Abdul Khudus, et al., Opt. Lett., 40(7):1318 (2015). 